\title{
Endometrial sampling in low-risk patients with abnormal uterine bleeding: a systematic review and meta-synthesis
}

\author{
Brenda F. Narice ${ }^{1 *}$ (D) Brigitte Delaney ${ }^{2}$ and Jon M. Dickson ${ }^{2}$
}

\begin{abstract}
Background: One million women per year seek medical advice for abnormal uterine bleeding (AUB) in the United Kingdom. Many low-risk patients who could be managed exclusively in primary care are referred to hospital based gynaecology services. Performing endometrial sampling (ES) in the community may improve care, reduce the rate of referrals and minimise costs. We aimed to search and synthesise the literature on the effectiveness of ES (Pipelle versus other devices) in managing AUB in low-risk patients.

Methods: We undertook an electronic literature search in MEDLINE via OvidSP, Scopus, and Web of Science for relevant English-language articles from 1984 to 2016 using a combination of MeSH and keywords. Two reviewers independently pre-selected 317 articles and agreed on 60 articles reporting data from over 7300 patients. Five themes were identified: sample adequacy, test performance, pain and discomfort, cost-effectiveness, and barriers and complications of office ES.

Results: Pipelle seems to perform as well as dilation and curettage and, as well or better than other ES devices in terms of sampling adequacy and sensitivity. It also seems to be better regarding pain/discomfort and costs. However, Pipelle can disrupt the sonographic appearance of the endometrium and may be limited by cervical stenosis, pelvic organ prolapse and endometrial atrophy.

Conclusions: The current evidence supports the use of Pipelle in the management of low-risk women presenting in the outpatient setting with symptomatic AUB when combined with clinical assessment and ultrasound scanning. However, the implications of its widespread use in primary care are uncertain and more research is required.
\end{abstract}

Keywords: Pipelle, Endometrial sampling, Abnormal uterine bleeding, Endometrial cancer, Endometrial hyperplasia, Premenopausal, Perimenopausal, Dilation and curettage

\section{Background}

Abnormal uterine bleeding (AUB), traditionally defined as uterine bleeding that is abnormal in volume, regularity, and/or timing [1] is common and affects $14-25 \%$ of women of reproductive age [2-4]. In the UK, approximately 1 million women seek medical advice for AUB every year, mostly in general practice $[5,6]$ and even though most cases could potentially be managed exclusively in primary care $[7,8]$, AUB is the fourth most common reason for referral to UK gynaecological services $[6,9,10]$. AUB has a major impact

\footnotetext{
* Correspondence: bnarice1@sheffield.ac.uk

${ }^{1}$ Clinical Research Fellow in Obstetrics \& Gynaecology; Academic Unit of Reproductive and Developmental Unit, University of Sheffield, Sheffield S10 2SF, UK

Full list of author information is available at the end of the article
}

on quality of life [7], leads to 3.5 million days of work absence [11], and generates significant health care costs. Hospital referrals and hysterectomies are the major components of the $£ 65$ million/year treatment costs for AUB [10].

Most cases of AUB are benign and amenable to office-based treatments $[12,13]$. However, patients often present with a myriad of symptoms, and their assessment requires training and expertise $[13,14]$. The causes of AUB can be summarised using the PALM-COEIN acronym: polyps, adenomyosis, leiomyoma/fibroids, malignancy (and hyperplasia), coagulopathy, ovulatory disorders, endometrial, iatrogenic, and not otherwise classified [1].

(C) The Author(s). 2018 Open Access This article is distributed under the terms of the Creative Commons Attribution 4.0 International License (http://creativecommons.org/licenses/by/4.0/), which permits unrestricted use, distribution, and 
Some patients who present with AUB will have endometrial hyperplasia or cancer which is the commonest gynaecological malignancy in the Western world. Even though the incidence rises after menopause, it can occur at all ages and $7 \%$ of cases are under $50[15,16]$. This percentage seems to be rising with increasing prevalence of obesity and diabetes $[17,18]$.

In the UK, women with AUB who are deemed at high risk of endometrial cancer such as those with postmenopausal bleeding (PMB) or family history of gynaecological neoplasms, should be referred to secondary care [19]. For low-risk premenopausal women the guidance is not as clear. Although urgent referral is not required [20], national guidelines recommend that endometrial sampling (ES) should be performed in women over 4045 years to exclude cancer [21, 22], but they do not specify whether ES should be performed in primary or secondary care [22].

In the UK, ES for AUB patients has not been traditionally undertaken in primary care. For many years, the standard management was dilation and curettage (D\&C) in hospital under general anaesthesia [23-25]. However, the need for admission and the risks of perforation and haemorrhage made D\&C unpopular [23, 25] and various ES devices were developed such as the Novak (a silastic cannula with a bevelled lateral opening [26]), the Tis-u-Trap (a plastic curette with suction [27]), the Vabra Aspirator (a stainless steel cannula connected to a vacuum pump [28]), the Endorette (a plastic cannula with multiple openings [29]), the Tao Brush (a sheath brush device [30]), the Cytospat (a polypropylene cannula with a rhomboid head [31]), the Accurette (a quadrilateral-shaped curette with four cutting edges [32]) and the Pipelle, the most widely used device in the UK (a flexible plastic tube with a distal circular port [27]).

We conducted a systematic review of the literature to identify existing evidence about the effectiveness of Pipelle compared with other ES techniques for assessing low-risk women with AUB which could inform the development of new care pathways in primary care.

\section{Why this study was necessary}

Endometrial sampling is thought to be a safe and effective method for histological assessment of the endometrium. It is used as an alternative to the more invasive method of $\mathrm{D} \& \mathrm{C}$. This is the first review to focus on AUB in low-risk pre- and perimenopausal women. We conclude that ES is a valuable tool in the assessment of these patients and that Pipelle is the best outpatient device available. The evidence supports the use of Pipelle in the outpatient setting but more research is required to assess its impact if introduced as routine management of AUB in the community.

\section{Methods}

\section{Literature search}

We used the PICO approach to develop a systematic search strategy [33]. We searched MEDLINE via OvidSP, Scopus, and Web of Science. For Medline, key concepts were identified (endometrial hyperplasia/cancer, abnormal uterine bleeding, endometrial sampling), a list of synonyms was generated for each concept and these lists were used to identify $\mathrm{MeSH}$ terms for the search (Additional file 1). Similar search strategies were used for Scopus and Web of Science (Additional file 1), always limited to papers from 1984 (when Pipelle was first introduced [34]) to 2016, written in English and involving humans.

We included papers investigating ES in women with AUB. We also considered studies in patients with known cancer; although these studies do not inform the indication of ES in primary care, they were an important source to evaluate test performance. We included review articles and opinion pieces. We excluded papers exclusively analysing postmenopausal patients, papers where the indication was assessment of fertility or recurrent miscarriage and papers where ES was assisted by hysteroscopy (unless this was used as a comparator to blind ES).

The initial search generated 173 results for Medline, 240 for Scopus, and 221 for Web of Science, totalling 634 search hits across all databases, 317 of which were excluded for duplication. The remaining 317 articles were assessed for inclusion using the titles and abstracts. The assessment was independently repeated by a second reviewer and a consensus was reached. After this process, 257 papers were excluded and the full text of 60 papers were read. Twenty-two further papers were excluded while another 22 papers were added from reference search, giving a final list of 60 papers. This selection included 16 randomized controlled trials (RCT), 26 prospective studies, 6 retrospective studies, 5 reviews, 2 meta-analyses, 1 survey, and 4 brief communications and letters to the editor, which were included in the final analysis providing data over 7300 women (Fig. 1).

\section{Bias risk assessment}

The quality of the RCTs was assessed using the standard Cochrane Risk of Bias tool [35], and the quality of observational studies was analysed with the modified Agency for Healthcare Research and Quality (AHRQ) quality assessment criteria [36].

\section{Results}

\section{Risk of Bias / quality of studies}

The overall quality of the RCTs was poor $(n=4)$ to moderate $(n=12)$, no high quality studies were identified. For observational studies, the risk of bias ranged from 31 to $79 \%$ with a mean weighted score $52.8 \% \mathrm{SD} \pm 11.8 \%$ which again suggests overall moderate quality [37]. See 


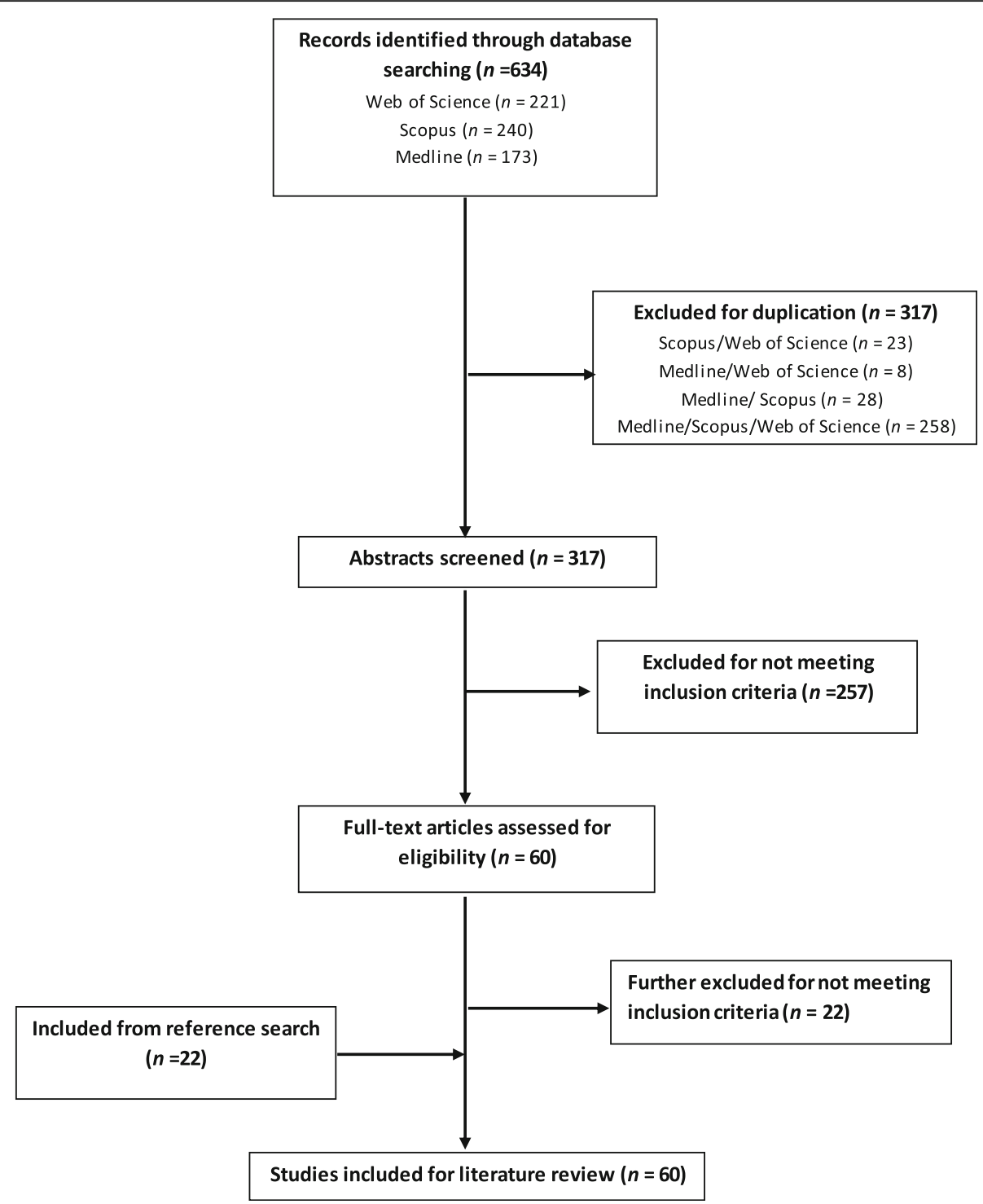

Fig. 1 PRISMA flow diagram for study selection

additional online content for tabulated assessments of individual studies (Additional file 2: TableS1 and 2, Additional file 2).

\section{Five themes}

We identified five major themes in the literature: (1) sample adequacy (defined as enough tissue to be analysed by pathologists [38]); (2) test performance when compared with hysterectomy and D\&C; (3) acceptability by the patient in terms of pain experienced during sampling; (4) the costs of taking outpatient endometrial biopsies; and (5) the barriers and complications of performing office ES.

All studies, except for one, were carried out in specialised outpatient gynaecology clinics or hospital services (secondary care) [39]. Only one study looked exclusively at premenopausal women [40]. The rest reported on cohorts of both pre- and post-menopausal women or they did not present results based on menopausal status. Most studies included women with symptomatic AUB and no risk of endometrial carcinoma. However, five studies targeted women with endometrial cancer to correlate pre-operative Pipelle with the hysterectomy histopathology [41-45]. Studies are summarised in Table 1.

\section{Sample adequacy}

Overall, the literature showed that the adequacy of material retrieved for histological analysis with Pipelle was comparable to $\mathrm{D} \& \mathrm{C}$ and superior to most of the other devices in pre-menopausal women. Ten studies [23, 24, 46-53] assessed Pipelle against D\&C in premenopausal women, reporting rates of adequacy ranging from $89.74 \%$ [51] to $98 \%[23,24]$ (Table 1). 
Table 1 Comparison of the RCTs, prospective and retrospective studies included in this literature review. Papers have been grouped by intervention/ comparator

\begin{tabular}{|c|c|c|c|c|c|c|}
\hline Study & Type of study & $\begin{array}{l}\text { Age of participants } \\
\text { (mean } \pm S D)\end{array}$ & $\begin{array}{l}\text { Intervention (n) vs } \\
\text { Comparator (n) }\end{array}$ & Outcome & Pain & Cost \\
\hline \multicolumn{7}{|l|}{$\begin{array}{l}\text { Pipelle versus } \\
\text { D\&C }+/- \\
\text { Hysterectomy }\end{array}$} \\
\hline $\begin{array}{l}\text { [23] Rauf } \\
\text { et al. } \\
\text { Pakistan } \\
2014\end{array}$ & $\mathrm{RCT}$ & $46.3 \pm 4.45$ & $\begin{array}{l}\text { Pipelle (102) vs } \\
\text { D\&C (101) }\end{array}$ & $\begin{array}{l}\text { Adequacy } \\
\text { Pipelle 98\% } \\
\text { D\&C 100\% }\end{array}$ & $\begin{array}{l}\text { Pipelle less } \\
\text { painful }\end{array}$ & $\begin{array}{l}\text { Pipelle } \\
\text { cheaper }\end{array}$ \\
\hline $\begin{array}{l}\text { [46] Liu et al. } \\
\text { China } 2015\end{array}$ & $\begin{array}{l}\text { Prospective } \\
\text { Sequential }\end{array}$ & 43.6 & $\begin{array}{l}\text { Pipelle vs D\&C } \\
\text { (245) }\end{array}$ & $\begin{array}{l}\text { Adequacy } \\
\text { Pipelle } 91.02 \% \\
\text { D\&C } 92.24 \%\end{array}$ & $\begin{array}{l}\text { Pipelle less } \\
\text { painful }\end{array}$ & N/A \\
\hline $\begin{array}{l}{[47]} \\
\text { Gungorduk } \\
\text { et al. Turkey } \\
2013\end{array}$ & Prospective & $\begin{array}{l}\text { Pipelle: } 49.8 \pm 6.1 \\
\text { D\&C: } 48.2 \pm 6.5\end{array}$ & $\begin{array}{l}\text { Pipelle + } \\
\text { hysterectomy } \\
\text { (78) vs D\&C+ } \\
\text { hysterectomy } \\
\text { (189) }\end{array}$ & $\begin{array}{l}\text { Adequacy } \\
\text { Pipelle 95\% } \\
\text { D\&C 96\% } \\
\text { Concordance } \\
\text { Pipelle + } \\
\text { hysterectomy } \\
62 \% \\
\text { D\&C+ } \\
\text { hysterectomy } \\
67 \%\end{array}$ & $\begin{array}{l}\text { Pipelle less } \\
\text { painful }\end{array}$ & $\begin{array}{l}\text { Pipelle } \\
\text { cheaper }\end{array}$ \\
\hline $\begin{array}{l}\text { [48] Kazandi } \\
\text { et al. } \\
\text { Turkey } \\
2012\end{array}$ & $\begin{array}{l}\text { Prospective } \\
\text { Sequential }\end{array}$ & $48 \pm 9.43$ & $\begin{array}{l}\text { Pipelle }+ \\
\text { hysterectomy } \\
\text { Vs } \\
\text { D\&C + } \\
\text { hysterectomy } \\
\text { (66) }\end{array}$ & $\begin{array}{l}\text { Adequacy } \\
\text { Pipelle 93\% } \\
\text { D\&C 96\% } \\
\text { Concordance } \\
\text { Pipelle and } \\
\text { D\&C 66\% } \\
\text { Pipelle \& } \\
\text { hysterectomy } \\
64 \%\end{array}$ & $\begin{array}{l}\text { Pipelle less } \\
\text { painful }\end{array}$ & $\begin{array}{l}\text { Pipelle } \\
\text { cheaper }\end{array}$ \\
\hline $\begin{array}{l}\text { [49] Demirkiran } \\
\text { et al. Turkey } 2012\end{array}$ & Prospective & 45.3 & $\begin{array}{l}\text { Pipelle + } \\
\text { hysterectomy } \\
\text { (212) vs D\&C+ } \\
\text { hysterectomy } \\
(161)\end{array}$ & $\begin{array}{l}\text { Adequacy } \\
\text { Pipelle 97\% } \\
\text { D\&C98\% } \\
\text { Concordance } \\
\text { Pipelle and } \\
\text { D\&C 84\% } \\
\text { Pipelle \& } \\
\text { hysterectomy } \\
67 \% \\
\text { D\&C and } \\
\text { hysterectomy } \\
\text { 80\% }\end{array}$ & $\begin{array}{l}\text { Pipelle less } \\
\text { painful }\end{array}$ & $\begin{array}{l}\text { Pipelle } \\
\text { cheaper }\end{array}$ \\
\hline $\begin{array}{l}\text { [43] Sany et al. } \\
\text { UK } 2011\end{array}$ & Retrospective & $?$ & $\begin{array}{l}\text { Pipelle + } \\
\text { hysterectomy } \\
\text { vs D\&C + } \\
\text { hysterectomy } \\
\text { (total 191) }\end{array}$ & $\begin{array}{l}\text { Concordance } \\
\text { Both techniques } \\
78 \%\end{array}$ & $\mathrm{~N} / \mathrm{A}$ & N/A \\
\hline $\begin{array}{l}\text { [45] Daud et al. } \\
\text { UK } 2011\end{array}$ & Retrospective & $55.7 \pm 11.4$ & $\begin{array}{l}\text { Pipelle } \pm \\
\text { hysterectomy } \\
\text { (75) vs D\&C } \pm \\
\text { hysterectomy } \\
(220)\end{array}$ & $\begin{array}{l}\text { Concordance } \\
\text { Pipelle + } \\
\text { hysterectomy } \\
76 \% \\
\text { D\&C+ } \\
\text { hysterectomy } \\
86 \%\end{array}$ & N/A & N/A \\
\hline $\begin{array}{l}\text { [24] Fakhar et al. } \\
\text { Pakistan } 2008\end{array}$ & $\begin{array}{l}\text { Prospective } \\
\text { Sequential }\end{array}$ & $45.4 \pm 7.2$ & $\begin{array}{l}\text { Pipelle versus } \\
\text { (D\&C) (100) }\end{array}$ & $\begin{array}{l}\text { Adequacy } \\
\text { Pipelle } 98 \% \\
\text { D\&C 100\% } \\
\text { NPV for } \\
\text { endometrial } \\
\text { carcinoma } \\
\text { Pipelle 100\% }\end{array}$ & $\begin{array}{l}\text { N/A (both } \\
\text { techniques } \\
\text { under GA) }\end{array}$ & $\begin{array}{l}\text { Pipelle } \\
\text { cheaper }\end{array}$ \\
\hline [44] Huang & Retrospective & $?$ & Pipelle + & Concordance & N/A & N/A \\
\hline
\end{tabular}


Table 1 Comparison of the RCTs, prospective and retrospective studies included in this literature review. Papers have been grouped by intervention/ comparator (Continued)

\begin{tabular}{|c|c|c|c|c|c|c|}
\hline Study & Type of study & $\begin{array}{l}\text { Age of participants } \\
\text { (mean } \pm \mathrm{SD} \text { ) }\end{array}$ & $\begin{array}{l}\text { Intervention (n) vs } \\
\text { Comparator (n) }\end{array}$ & Outcome & Pain & Cost \\
\hline et al. USA 2006 & \multirow[t]{2}{*}{ + Letter } & & \multirow{2}{*}{$\begin{array}{l}\text { hysterectomy } \\
(253) \text { vs D\&C + } \\
\text { hysterectomy } \\
\text { (93) }\end{array}$} & \multirow[b]{2}{*}{$\begin{array}{l}\text { Pipelle and } \\
\text { hysterectomy } \\
93.8 \% \text { (low grade } \\
\text { cancer) \& } 99.2 \% \\
\text { (high grade cancer) } \\
\text { D\&C and } \\
\text { hysterectomy } \\
97 \% \text { (low grade } \\
\text { cancer) } \\
\& 100 \% \text { (high } \\
\text { grade cancer) }\end{array}$} & & \\
\hline $\begin{array}{l}\text { [37] Macones } \\
\text { et al. } 2006\end{array}$ & & & & & & \\
\hline $\begin{array}{l}\text { [66] Machado } \\
\text { et al. Spain } \\
2002\end{array}$ & Retrospective & $\begin{array}{l}\text { Post-menopausal } \\
(68) \\
\text { Pre- or } \\
\text { peri-menopausal } \\
(100)\end{array}$ & $\begin{array}{l}\text { Pipelle (168) } \\
\text { vs D\&C (92) } \pm \\
\text { Hysterectomy } \\
(76)\end{array}$ & $\begin{array}{l}\text { Accuracy } \\
\text { Sensitivity } 84.2 \% \\
\text { Specificity } 99.1 \%\end{array}$ & $\mathrm{~N} / \mathrm{A}$ & N/A \\
\hline $\begin{array}{l}\text { [51] Kavak et al. } \\
\text { Turkey } 1996\end{array}$ & Prospective & $50.8 \pm 7.8$ & $\begin{array}{l}\text { Pipelle } \pm \text { TVS } \\
\text { (78) vs D\&C } \\
\text { (78) }\end{array}$ & $\begin{array}{l}\text { Concordance } \\
\text { Sensitivity: } 73 \% \\
\text { (increased to } 90 \% \\
\text { with TVS) } \\
\text { Specificity: } 100 \%\end{array}$ & N/A & $\mathrm{N} / \mathrm{A}$ \\
\hline $\begin{array}{l}\text { [50] Ben-Baruch } \\
\text { et al. Israel } 1993\end{array}$ & Prospective & $\begin{array}{l}\text { Pre- and } \\
\text { post-menopausal }\end{array}$ & $\begin{array}{l}\text { Pipelle (172) } \\
\text { vs D\&C (97) }\end{array}$ & $\begin{array}{l}\text { Adequacy } \\
\text { Pipelle } 90.6 \% \\
\text { D\&C 68\% }\end{array}$ & $\mathrm{N} / \mathrm{A}$ & $\mathrm{N} / \mathrm{A}$ \\
\hline $\begin{array}{l}\text { [68] Sanam } \\
\text { et al. Iran } 2015\end{array}$ & Prospective & $>35$ & $\begin{array}{l}\text { Pipelle (130) } \\
\text { vs D\&C (130) }\end{array}$ & $\begin{array}{l}\text { Concordance } \\
\text { Pipelle and } \\
\text { D\&C 94\% } \\
\text { Adequacy } \\
\text { Pipelle } 84.6 \% \\
\text { D\&C 90\% }\end{array}$ & $\mathrm{N} / \mathrm{A}$ & $\begin{array}{l}\text { Pipelle } \\
\text { cheaper }\end{array}$ \\
\hline $\begin{array}{l}\text { [75] Gordon } \\
\text { New Zealand } \\
1999\end{array}$ & Prospective & $47.2 \pm 1.8$ & $\begin{array}{l}\text { Pipelle (100) } \\
\text { vs D\&C or } \\
\text { hysterectomy } \\
(n=?)\end{array}$ & $\begin{array}{l}\text { Adequacy } \\
\text { Pipelle } 67 \%\end{array}$ & N/A & N/A \\
\hline $\begin{array}{l}\text { [69] Goldchmit } \\
\text { et al. Israel } \\
1993\end{array}$ & $\begin{array}{l}\text { Prospective } \\
\text { Sequential }\end{array}$ & 48.1 & $\begin{array}{l}\text { Pipelle and TVS } \\
\text { vs D\&C (176) }\end{array}$ & $\begin{array}{l}\text { Concordance } \\
\text { Pipelle \& D\&C } \\
90 \% \text { (increased } \\
\text { to } 92 \% \text { with TVS) }\end{array}$ & N/A & $\mathrm{N} / \mathrm{A}$ \\
\hline $\begin{array}{l}\text { [52] Abdelazim } \\
\text { et al. Turkey } \\
2013\end{array}$ & $\begin{array}{l}\text { Prospective } \\
\text { Sequential }\end{array}$ & 44.5 & $\begin{array}{l}\text { Pipelle vs } \\
\text { D\&C (143) }\end{array}$ & $\begin{array}{l}\text { Adequacy } \\
\text { Pipelle 97.9\% } \\
\text { D\&C 100\% } \\
\text { NPV for } \\
\text { endometrial } \\
\text { polyp } \\
\text { Pipelle 89.6\% }\end{array}$ & $\mathrm{N} / \mathrm{A}$ & $\mathrm{N} / \mathrm{A}$ \\
\hline $\begin{array}{l}\text { [72] Shams } \\
\text { Pakistan } 2012\end{array}$ & $\begin{array}{l}\text { Prospective } \\
\text { Sequential }\end{array}$ & 47.94 & $\begin{array}{l}\text { Pipelle vs } \\
\text { D\&C (50) }\end{array}$ & $\mathrm{N} / \mathrm{A}$ & $\begin{array}{l}\text { Pipelle less } \\
\text { painful }\end{array}$ & $\begin{array}{l}\text { Pipelle } \\
\text { cheaper }\end{array}$ \\
\hline $\begin{array}{l}\text { [53] Rezk et al. } \\
\text { Egypt } 2016\end{array}$ & Prospective & $\begin{array}{l}\text { Pipelle: } 47.2 \pm 3.8 \\
\text { D\&C: } 46.9 \pm 4.1\end{array}$ & $\begin{array}{l}\text { Pipelle (270) } \\
\text { vs D\&C (268) }\end{array}$ & $\begin{array}{l}\text { Adequacy } \\
\text { No difference } \\
\left(p^{>} 0.05\right)\end{array}$ & $\begin{array}{l}\text { D\&C less } \\
\text { painful }\end{array}$ & N/A \\
\hline \multicolumn{7}{|l|}{$\begin{array}{l}\text { Pipelle versus } \\
\text { Vabra }+/- \\
\text { Hysterectomy }\end{array}$} \\
\hline $\begin{array}{l}\text { [54] Eddowes } \\
\text { et al. UK } 1990\end{array}$ & $\begin{array}{l}\text { Prospective } \\
\text { Sequential }\end{array}$ & 41.6 & $\begin{array}{l}\text { Pipelle vs Vabra } \\
\text { Aspirator (100) }\end{array}$ & $\begin{array}{l}\text { Adequacy } \\
\text { Pipelle 88\% } \\
\text { Vabra Aspirator } \\
88 \%\end{array}$ & $\begin{array}{l}\text { Pipelle less } \\
\text { painful }\end{array}$ & $\begin{array}{l}\text { Pipelle } \\
\text { cheaper }\end{array}$ \\
\hline $\begin{array}{l}\text { [55] Naim et al. } \\
\text { Malaysia } 2007\end{array}$ & $\mathrm{RCT}$ & $>45$ & $\begin{array}{l}\text { Pipelle (76) vs } \\
\text { Vabra Aspirator }\end{array}$ & $\begin{array}{l}\text { Adequacy } \\
\text { Pipelle } 73.3 \%\end{array}$ & $\mathrm{~N} / \mathrm{A}$ & $\begin{array}{l}\text { Pipellle } \\
\text { cheaper }\end{array}$ \\
\hline
\end{tabular}


Table 1 Comparison of the RCTs, prospective and retrospective studies included in this literature review. Papers have been grouped by intervention/ comparator (Continued)

\begin{tabular}{|c|c|c|c|c|c|c|}
\hline Study & Type of study & $\begin{array}{l}\text { Age of participants } \\
\text { (mean } \pm \text { SD) }\end{array}$ & $\begin{array}{l}\text { Intervention (n) vs } \\
\text { Comparator (n) }\end{array}$ & Outcome & Pain & Cost \\
\hline & & & (71) & Vabra 52.4\% & & \\
\hline $\begin{array}{l}\text { [28] Kaunitz } \\
\text { et al. USA } \\
1988\end{array}$ & $\begin{array}{l}\text { Prospective } \\
\text { Sequential }\end{array}$ & 46 & $\begin{array}{l}\text { Pipelle vs } \\
\text { Vabra (56) }\end{array}$ & $\begin{array}{l}\text { Adequacy } \\
\text { Pipelle \& Vabra } \\
91 \% \\
\text { Concordance } \\
\text { Pipelle \& Vabra } \\
89 \%\end{array}$ & $\begin{array}{l}\text { Pipelle less } \\
\text { painful }\end{array}$ & $\begin{array}{l}\text { Pipelle } \\
\text { cheaper }\end{array}$ \\
\hline $\begin{array}{l}\text { [56] Rodriguez } \\
\text { et al. USA } 1993\end{array}$ & $\mathrm{RCT}$ & $?$ & $\begin{array}{l}\text { Pipelle (12) vs } \\
\text { Vabra (13) vs } \\
\text { Hysterectomy } \\
(25)\end{array}$ & $\begin{array}{l}\text { Surface being } \\
\text { sampled: } \\
\text { Pipelle } 4.2 \% \\
\text { Vabra } 41.6 \%\end{array}$ & $\mathrm{~N} / \mathrm{A}$ & $\mathrm{N} / \mathrm{A}$ \\
\hline \multicolumn{7}{|l|}{$\begin{array}{l}\text { Pipelle versus } \\
\text { Tao Brush+/- } \\
\text { Hysteroscopy }\end{array}$} \\
\hline $\begin{array}{l}\text { [30] Williams } \\
\text { et al. UK } 2008\end{array}$ & RCT Sequential & $\begin{array}{l}\text { Moderate risk: } \\
45.2 \text { (SE 0.26) }\end{array}$ & $\begin{array}{l}\text { For moderate } \\
\text { risk Pipelle (34) } \\
\text { Tao Brush (29) }\end{array}$ & $\begin{array}{l}\text { Adequacy } \\
\text { Both techniques } \\
84 \% \\
\text { No significant } \\
\text { difference for } \\
\text { premenopausal }\end{array}$ & $\begin{array}{l}\text { Tao Brush } \\
\text { less painful }\end{array}$ & N/A \\
\hline $\begin{array}{l}\text { [57] Critchley } \\
\text { et al. UK } 2004\end{array}$ & RCT & $\begin{array}{l}\text { Moderate risk: } \\
\text { pre-menopausal } \\
\text { >40 or }<40 \text { with } \\
\text { risk for } \\
\text { endometrial } \\
\text { cancer } \\
\text { Low risk }\end{array}$ & $\begin{array}{l}\text { Pipelle vs Tao } \\
\text { Brush } \\
\text { Moderate risk } \\
\text { (Total 326) } \\
\text { Low risk } \\
\text { (Total 157) } \\
\pm \text { hysteroscopy } \\
\pm \text { TVS }\end{array}$ & $\begin{array}{l}\text { Successful } \\
\text { completion of } \\
\text { investigation: } \\
\text { Pipelle } 85 \% \\
\text { Adequacy of } \\
\text { sample with } \\
\text { Pipelle: } \\
\text { Moderate } \\
\text { risk } 79 \%\end{array}$ & $\begin{array}{l}\text { Tao Brush } \\
\text { less painful } \\
\text { than Pipelle }\end{array}$ & $\begin{array}{l}\text { Minimal } \\
\text { difference }\end{array}$ \\
\hline $\begin{array}{l}\text { [58] Yang et al. } \\
\text { USA } 2003\end{array}$ & $\begin{array}{l}\text { Prospective } \\
\text { Sequential }\end{array}$ & $24-86$ & $\begin{array}{l}\text { Pipelle (79) vs } \\
\text { Tao Brush (79) }\end{array}$ & $\begin{array}{l}\text { Factors affecting } \\
\text { sensitivity: tumour } \\
\text { size, type, location } \\
\text { within the uterus, } \\
\text { sampling } \\
\text { mechanism and } \\
\text { preparation } \\
\text { method }\end{array}$ & N/A & N/A \\
\hline $\begin{array}{l}\text { [59] Del Priore } \\
\text { et al. USA } 2001\end{array}$ & RCT Sequential & $\begin{array}{l}\text { Pre-menopausal: } \\
46 \\
\text { Post-menopausal: } \\
61\end{array}$ & $\begin{array}{l}\text { Tao Brush vs } \\
\text { Pipelle (50) }\end{array}$ & $\begin{array}{l}\text { Sensitivity: } \\
\text { Pipelle } 86 \% \\
\text { Tao Brush 95.5\% } \\
\text { Specificity: } \\
\text { Both 100\% }\end{array}$ & N/A & $\begin{array}{l}\text { Tao Brush } \\
\text { cheaper } \\
\text { than D\&C }\end{array}$ \\
\hline $\begin{array}{l}\text { [60] Yang et al. } \\
\text { USA } 2000\end{array}$ & $\begin{array}{l}\text { Prospective } \\
\text { Sequential }\end{array}$ & 58 & $\begin{array}{l}\text { Tao Brush vs } \\
\text { Tao Brush + } \\
\text { Pipelle (25) }\end{array}$ & $\begin{array}{l}\text { Adequacy } \\
\text { Tao Brush 98\% } \\
\text { Pipelle 88\% }\end{array}$ & $\begin{array}{l}\text { Tao Brush } \\
\text { less painful }\end{array}$ & Comparable \\
\hline \multicolumn{7}{|l|}{$\begin{array}{l}\text { Pipelle versus } \\
\text { Novak }\end{array}$} \\
\hline $\begin{array}{l}\text { [40] Henig et al. } \\
\text { USA } 1989\end{array}$ & $\mathrm{RCT}$ & Pre-menopausal & $\begin{array}{l}\text { Pipelle (50) } \\
\text { Vs Novak (50) }\end{array}$ & $\begin{array}{l}\text { Adequacy } \\
\text { Pipelle 94\% } \\
\text { Novak 98\% }\end{array}$ & $\begin{array}{l}\text { Better tolerance } \\
\text { with Pipelle }\end{array}$ & N/A \\
\hline $\begin{array}{l}\text { [26] Stovall et al. } \\
\text { USA } 1991\end{array}$ & $\mathrm{RCT}$ & $\begin{array}{l}\text { Pipelle: } 40 \\
\text { Novak: } 44\end{array}$ & $\begin{array}{l}\text { Pipelle (149) } \\
\text { vs Novak (126) }\end{array}$ & $\begin{array}{l}\text { Adequacy } \\
\text { Pipelle } 87.2 \% \\
\text { Novak } 90.5 \%\end{array}$ & $\begin{array}{l}\text { Pipelle less } \\
\text { painful }\end{array}$ & $\begin{array}{l}\text { Novak might } \\
\text { be cheaper }\end{array}$ \\
\hline $\begin{array}{l}\text { [61] Silver et al. } \\
\text { USA } 1991\end{array}$ & $\begin{array}{l}\text { RCT } \\
\text { Sequential }\end{array}$ & $28-76$ & $\begin{array}{l}\text { 1st Pipelle then } \\
\text { Novak (26) vs } \\
\text { 1st Novak then } \\
\text { Pipelle (29) }\end{array}$ & $\begin{array}{l}\text { Adequacy } \\
\text { Similar }\end{array}$ & $\begin{array}{l}\text { Pipelle less } \\
\text { painful }\end{array}$ & N/A \\
\hline
\end{tabular}


Table 1 Comparison of the RCTs, prospective and retrospective studies included in this literature review. Papers have been grouped by intervention/ comparator (Continued)

\begin{tabular}{|c|c|c|c|c|c|c|}
\hline Study & Type of study & $\begin{array}{l}\text { Age of participants } \\
\text { (mean } \pm \text { SD) }\end{array}$ & $\begin{array}{l}\text { Intervention (n) vs } \\
\text { Comparator (n) }\end{array}$ & Outcome & Pain & Cost \\
\hline $\begin{array}{l}\text { [67] Guido } \\
\text { et al. USA } \\
1995\end{array}$ & $\begin{array}{l}\text { Prospective } \\
\text { Sequential }\end{array}$ & 61 & $\begin{array}{l}\text { Pipelle vs } \\
\text { Hysterectomy } \\
(71)\end{array}$ & $\begin{array}{l}\text { Adequacy } \\
\text { Pipelle } 97 \% \\
\text { Concordance } \\
\text { Pipelle \& } \\
\text { hysterectomy } \\
83 \%\end{array}$ & N/A & N/A \\
\hline $\begin{array}{l}\text { [42] Ferry } \\
\text { et al. UK } \\
1993\end{array}$ & $\begin{array}{l}\text { Prospective } \\
\text { Sequential }\end{array}$ & $?$ & $\begin{array}{l}\text { Pipelle vs } \\
\text { Hysterectomy } \\
\text { (37) }\end{array}$ & $\begin{array}{l}\text { Concordance } \\
\text { Pipelle \& } \\
\text { hysterectomy } \\
67 \%\end{array}$ & N/A & N/A \\
\hline $\begin{array}{l}\text { [41] G Zorlu } \\
\text { et al. Turkey } \\
1994\end{array}$ & $\begin{array}{l}\text { Prospective } \\
\text { Sequential }\end{array}$ & 51 & $\begin{array}{l}\text { Pipelle vs } \\
\text { Hysterectomy } \\
\text { (26) }\end{array}$ & $\begin{array}{l}\text { Concordance } \\
\text { Pipelle \& } \\
\text { hysterectomy } \\
95 \%\end{array}$ & $\begin{array}{l}\text { Mild pain } \\
\text { and } \\
\text { discomfort } \\
\text { with Pipelle }\end{array}$ & N/A \\
\hline
\end{tabular}

Pipelle versus

Explora $+/-$

Accurette

\section{[62] Leclair \\ et al. USA \\ 2011}

[32] Lipscomb et al. USA

1994

Pipelle versus Infant Feeding Tube (IFT)

[63] Bhide et al. UK 2007

Pipelle Mark 2 versus Pipelle Mark $2+$

hysteroscopy

\section{[71] Polena}

et al. France

2006

Prospective
Sequential

50

Pipelle: $45.2 \pm 7.3 \quad$ Pipelle (37) vs

Explora: $46.1 \pm 7.7$

N/A

Pre- and post-

menopausal

Accurette (81)

vs Explora (82)

Prospective

$?$

Pipelle (29)

vs IFT (31)

Pipelle Mark

2 vs Pipelle

Mark $2 \pm$

hysteroscopy

(97)

Pipelle: 42.9

Tis-u-trap: 42.3

Pipelle +

hysterectomy

(74) vs

Tis-u-trap +

hysterectomy

(75)
Adequacy

Pipelle 73\%

IFT $71 \%$
Less pain with IFT

$\begin{array}{ll}\text { Adequacy } & \text { No } \\ \text { Pipelle } 91 \% & \text { differences } \\ \text { Explora } 97 \% & \text { seen }\end{array}$

No

significant

difference in

pain score

N/A

N/A

Accurette $72.5 \%$

Explora 85.4\%
No difference

with

conventional

Pipelle

$88.7 \%$

Adequacy

Pipelle 87.8\%

Tis-u-trap 84\%

Concordance

Pipelle \&

hysterectomy

85\%

Tis-u-trap \&

hysterectomy

92\%

Pipelle versus Endorette

[29] Moberger

RCT

et al. Sweden

Sequential

$57.5 \pm 11.5$

Pipelle vs

Endorette

(152)

Adequacy and concordance No difference
No

significant

difference
Slightly more expensive

than

conventional Pipelle

Pipelle cheaper

Pipelle versus Cytospat +/- 
Table 1 Comparison of the RCTs, prospective and retrospective studies included in this literature review. Papers have been grouped by intervention/ comparator (Continued)

\begin{tabular}{|c|c|c|c|c|c|c|}
\hline Study & Type of study & $\begin{array}{l}\text { Age of participants } \\
\text { (mean } \pm \text { SD) }\end{array}$ & $\begin{array}{l}\text { Intervention (n) vs } \\
\text { Comparator (n) }\end{array}$ & Outcome & Pain & Cost \\
\hline \multicolumn{7}{|c|}{ Hysterectomy } \\
\hline $\begin{array}{l}\text { [31] Antoni } \\
\text { et al. Spain } \\
1996\end{array}$ & $\mathrm{RCT}$ & $48.6 \pm 9$ & $\begin{array}{l}\text { Pipelle } \pm \\
\text { hysterectomy } \\
\text { or D\&C (191) } \\
\text { vs Cytospat } \pm \\
\text { hysterectomy } \\
\text { or D\&C (174) }\end{array}$ & $\begin{array}{l}\text { Adequacy } \\
\text { Pipelle } 75 \% \\
\text { Cytospat } 76 \% \\
\text { Concordance } \\
\text { Pipelle: Benign } \\
\text { 84\%, Hyperplasia } \\
71 \% \text {, Malignancy } \\
60 \% \\
\text { Cytospat: Benign } \\
82 \% \text {, Hyperplasia } \\
60 \% \text {, Malignancy } \\
60 \%\end{array}$ & $\begin{array}{l}\text { Better } \\
\text { tolerance } \\
\text { for Pipelle }\end{array}$ & $\begin{array}{l}\text { Pipelle } \\
\text { cheaper }\end{array}$ \\
\hline
\end{tabular}

Pipelle versus

D\&C +/-

Hysteroscopy

+/- TV US

[85] Tahir

et al. UK

1999

[34] Cornier

France 1984

[74] Frishman

USA 1990

[38] Mc

Cluggage

Northern

Ireland 2006

[79] Van Den

Bosch Belgium

2005

[76] Brandner

Germany

2000

[80] Dijkhuizen

et al. The

Netherlands

2000

[25] Cooper

et al.

USA 2000
RCT

35

RCT

Anaesthesia

for Pipelle

Brief

communication

Lidocaine:

$42.1 \pm 11.9 /$

Saline:

$44.9 \pm 12.5$

Mostly

pre-menopausal

N/A

Letter in

Prospective

sequential

Pre-menopausal:

$41.6 \pm 8.7$

Post-menopausal:

$59 \pm 9.9$

Review

N/A

Inpatient: Adequacy

Hysteroscopy No difference

\& D\&C (200) Concordance

vs Outpatient: Inpatient: 100\&

Pipelle +/- TV Outpatient: 82\&

US +/-

Hysteroscpy

(200)

39 studies

including

7914 patients

Review

N/A
N/A

Lidocaine
(19) vs Saline
(22)
Pipelle (250)
No control

Pipelle versus

Tis-u-Trap

Pipelle versus

other ES

US before and after Pipelle (99)

N/A

Significant reduction of pain with lidocaine

Useful for

histologic

dating of the

endometrium

N/A

Difficulties of

processing

outpatient

ES samples

Thickness of the endometrium

ET on average

$0.4 \mathrm{~mm}$ less after performing

Pipelle

Progression of

endometrial

lesions (potential

limitations for ES)

Different ES

Pipelle is superior to other ES for diagnosing cancer/ hyperplasia

Directed biopsy with Hysteroscopy: most accurate ES (not for primary care)
More pain in N/A outpatient

N/A

with

intervention

Little

discomfort

Low cost

N/A

Pipelle cheaper

N/A

N/A

N/A

N/A

N/A

N/A

N/A

N/A

N/A

N/A 
Table 1 Comparison of the RCTs, prospective and retrospective studies included in this literature review. Papers have been grouped by intervention/ comparator (Continued)

\begin{tabular}{|c|c|c|c|c|c|c|}
\hline Study & Type of study & $\begin{array}{l}\text { Age of participants } \\
\text { (mean } \pm S D \text { ) }\end{array}$ & $\begin{array}{l}\text { Intervention (n) vs } \\
\text { Comparator (n) }\end{array}$ & Outcome & Pain & Cost \\
\hline $\begin{array}{l}\text { [14] Farquhar } \\
\text { et al. } \\
\text { New Zealand } 1996\end{array}$ & Survey & $\begin{array}{l}68 \text { replies from } \\
\text { O\&G consultants } \\
\text { (48\% of all } \\
\text { contestants) }\end{array}$ & N/A & $\begin{array}{l}\text { Management of } \\
\text { menorrhagia in } \\
\text { primary care }\end{array}$ & $\mathrm{N} / \mathrm{A}$ & $\mathrm{N} / \mathrm{A}$ \\
\hline $\begin{array}{l}\text { [78] Youssif et al. } \\
\text { Australia } 1995\end{array}$ & Review & N/A & N/A & $\begin{array}{l}\text { Effectiveness and } \\
\text { safety of Pipelle }\end{array}$ & $\mathrm{N} / \mathrm{A}$ & N/A \\
\hline $\begin{array}{l}\text { [77] Dantas et al. } \\
\text { Brazil } 1994\end{array}$ & Letter & $\begin{array}{l}\text { Nurses vs doctors } \\
\text { performing Pipelle }\end{array}$ & N/A & $\begin{array}{l}\text { Adequacy No } \\
\text { difference }\end{array}$ & $\mathrm{N} / \mathrm{A}$ & $\mathrm{N} / \mathrm{A}$ \\
\hline $\begin{array}{l}\text { [82] Clark et al. } \\
\text { UK } 2002\end{array}$ & $\begin{array}{l}\text { Systematic } \\
\text { review and } \\
\text { meta-analysis }\end{array}$ & $\begin{array}{l}\text { Mixed pre- }(21 \%) \\
\text { and pos- } \\
\text { tmenopausal }(79 \%)\end{array}$ & $\begin{array}{l}\text { Pipelle vs other } \\
\text { outpatient } \\
\text { techniques }\end{array}$ & $\begin{array}{l}\text { Likelihood ratio of } \\
\text { endometrial cancer } \\
\text { when Pipelle is: } \\
\text {-ve: } 0.1 \\
\text { +ve: } 64.6\end{array}$ & $\mathrm{~N} / \mathrm{A}$ & N/A \\
\hline $\begin{array}{l}\text { [86] Ahonkallio } \\
\text { et al. Finland } \\
2009\end{array}$ & Prospective & $\begin{array}{l}\text { Range } 47-52 \\
\text { Post ablation }\end{array}$ & Pipelle (57) & $\begin{array}{l}\text { Adequacy } \\
29 \% \text { failure If } \\
\text { endometrium } \\
<5 \mathrm{~mm} \\
5 \% \text { failure if } \\
\text { endometrium } \\
>5 \mathrm{~mm}\end{array}$ & $\mathrm{~N} / \mathrm{A}$ & N/A \\
\hline $\begin{array}{l}\text { [81] Du et al. } \\
\text { China } 2016\end{array}$ & Review & N/A & N/A & $\begin{array}{l}\text { Most appropriate } \\
\text { ES devices for } \\
\text { endometrial } \\
\text { lesions }\end{array}$ & $\begin{array}{l}\text { Little } \\
\text { discomfort }\end{array}$ & N/A \\
\hline $\begin{array}{l}\text { [64] Masood } \\
\text { et al. Pakistan } \\
2015\end{array}$ & $\begin{array}{l}\text { Cross } \\
\text { sectional }\end{array}$ & $\begin{array}{l}\text { Pre- and } \\
\text { post-menopausal } \\
35-48\end{array}$ & $\begin{array}{l}\text { Pipelle (126) } \\
\text { vs no } \\
\text { comparator }\end{array}$ & $\begin{array}{l}\text { Adequacy } \\
\text { Pipelle } 96.82 \%\end{array}$ & $\mathrm{~N} / \mathrm{A}$ & Cost-effective \\
\hline $\begin{array}{l}\text { [39] Seamark } \\
\text { UK } 1998\end{array}$ & Prospective & $\begin{array}{l}\geq 40 \\
42-74 \\
\text { Primary care } \\
\text { population }\end{array}$ & $\begin{array}{l}\text { Pipelle (38) vs } \\
\text { no comparator }\end{array}$ & $\begin{array}{l}\text { Adequacy } \\
\text { Pipelle } 76 \%\end{array}$ & $\mathrm{~N} / \mathrm{A}$ & N/A \\
\hline $\begin{array}{l}\text { [70]Seto } \\
\text { UK } 2016\end{array}$ & Retrospective & $\begin{array}{l}\text { Pre-menopausal } \\
46.1 \pm 4.6 \\
\text { Post-menopausal } \\
57.2 \pm 8.1\end{array}$ & $\begin{array}{l}\text { Pipelle against } \\
\text { hysteroscopy }\end{array}$ & $\begin{array}{l}\text { Positive predictive } \\
\text { value for } \\
\text { endometrial polyp } \\
\text { Pipelle } \\
\text { (pre-menopausal) } \\
53.7 \%\end{array}$ & $\mathrm{~N} / \mathrm{A}$ & $\mathrm{N} / \mathrm{A}$ \\
\hline $\begin{array}{l}\text { [65] Piatek } \\
\text { et al. Poland } \\
2016\end{array}$ & Retrospective & $\begin{array}{l}\text { Pre- and } \\
\text { post-menopausal }\end{array}$ & $\begin{array}{l}\text { Pipelle (312) vs } \\
\text { no comparator }\end{array}$ & $\begin{array}{l}\text { Adequacy } \\
83.01 \%\end{array}$ & $\mathrm{~N} / \mathrm{A}$ & $\mathrm{N} / \mathrm{A}$ \\
\hline
\end{tabular}

ES Endometrial sampling, AUB Abnormal uterine bleeding, RCT Randomized controlled trials, US Transvaginal ultrasound, N/A Non-applicable,? Unknown

Three studies compared the sample adequacy of Pipelle and Vabra Aspirator [28, 54, 55]. One of these studies [55] showed better rates for Pipelle (73.3\% versus $52.4 \%, P$ $=0.02$ ) whereas the remaining two could not identify any significant difference between both techniques (one study reported $91 \%$ for both techniques [28] whereas the other showed $89.79 \%$ for Vabra versus $88 \%$ for Pipelle [54], no $P$ values provided) (Table 1). We also found a RCT which reported that Pipelle despite being equal or superior to Vabra in terms of sample adequacy only assesses $4.2 \%$ of the endometrium versus $41.6 \%$ with Vabra [56].

Five studies including mixed cohorts of pre- and postmenopausal women compared sample adequacy between Pipelle and Tao Brush [30, 57-60]. Despite one study suggesting that Tao Brush bendable wire should improve sampling of the uterine lateral walls when compared to Pipelle more rigid structure, none of the studies showed significant differences in premenopausal populations [58] (Table 1).

Two studies [40,61] also compared Pipelle to Novak and found no statistically significant difference in terms of adequacy of sample, which varied from 83 to $94 \%$ for Pipelle and from 85 to $98 \%$ for Novak [40,61] (Table 1). Six additional studies did not find a significant difference when comparing Pipelle with other less popular ES techniques such as Explora [32, 62] (85.4-97\% for Explora versus $85.2-91 \%$ for Pipelle), Tis-u-trap [27] (88\% for Pipelle versus $84 \%$ for Tis-u-Trap $P=0.5$ ), Endorette 
[29] (56\% for Endorette versus 43\% for Pipelle), infant feeding tube [63] (73\% for Pipelle versus $71 \%$ for IFT) and Cytospat [31] (Pipelle 74.9\% versus 75.9\% for Cytospat).

Three studies [39, 64, 65] assessed the ability of Pipelle to retrieve enough tissue for histological analysis without comparing it to other devices, and reported a success rate of $76 \%$ in GP practices [39], and a range from 83.01 to $96.82 \%$ in secondary care $[64,65]$ (Table 1 ).

\section{Test performance}

Nine studies compared the histopathological diagnosis of pre-operative Pipelle and $D \& C$ with the final results from hysterectomy (the gold standard diagnostic technique for uterine disorders) [41, 43-45, 47-49, 66, 67]. For Pipelle, the sensitivity ranged from $62 \%$ [47] to $99.2 \%$ [44] and for D\&C sensitivity varied from $67 \%$ [47] to $100 \%$ [44]. One of these studies applied Pipelle and D\&C sequentially before hysterectomy [48], while the rest were multi-arm studies [41, 43-45, 47, 49, 66, 67] (Table 1).

At least 5 studies [43, 48, 49, 68, 69] also reported on the concordance between Pipelle and $D \& C$ with values that ranged from 66\% [48] to 94\% [68].

One retrospective study which compared Pipelle samples suggestive of endometrial polyps with subsequent hysteroscopically-guided polypectomies reported Pipelle had a positive predictive value of $55.3 \%$ for sampling polyps in premenopausal women [70]. Pipelle has also been reported to have $100 \%$ negative predictive value (NPV) for endometrial carcinoma and hyperplasia [24] and up to $99.2 \%$ NPV for endometritis and $89.6 \%$ for endometrial polyps [52] (Table 1).

\section{Pain / discomfort}

Most studies included in this review performed ES on awake patients, but only 23 studies formally assessed patients' pain using visual pain analogue scales and questionnaires (Table 1). A total of 15 studies reported that most patients experienced minimal discomfort with Pipelle [23, 26, 28, 31, 34, 40, 41, 46-49, 54, 61, 71, 72], three did not find any significant difference between Pipelle and Explora [32, 62] and Pipelle and Endorette [29], three concluded that Tao Brush was better tolerated than Pipelle $[30,57,60]$ and one study showed less discomfort when using an infant feeding tube as a prototype [63]. A RCT also reported that paracervical lidocaine during Pipelle may decrease pain when compared to placebo [73] (Table 1).

\section{Costs of outpatient endometrial sampling}

A total of 17 studies assessed the cost-effectiveness of Pipelle though none formally provided a health economic analysis $[23,24,26-28,31,47-49,54,55,57,60,68,71$, $72,74]$. Some of the factors they considered when assessing the total cost of ES were the need for general anaesthesia and hospital admission [23, 72] and the cost of operative hysteroscopy/ D\&C following a failed office ES or an inadequate sample [55]. Fifteen studies showed Pipelle was cheaper than the alternative ES $[23,24,26-28$, $31,47-49,54,55,57,68,72,74]$ and two did not find significant differences between Pipelle and Pipelle Mark 2 [71], and Pipelle and Tao Brush [60]. Two studies concluded that the Vabra was cheaper than Pipelle given its multiple use [26] but when all costs were considered including the need for follow-up for failed procedures, the average cost of Pipelle per patient was approximately 30\% cheaper than the Vabra aspirator [55] (Table 1).

\section{Barriers and complications to endometrial sampling in primary care}

Several limitations to successful ES were reported including cervical stenosis and pelvic organ prolapse which hindered the access to the uterine cavity $[24,75]$ as well as focal endometrial pathology (e.g. endometrial polyps and submucosal fibroids) and endometrial atrophy which reduces sample adequacy [30, 46, 69, 75, 76]. Lack of experience was also linked to inadequate sampling with higher failure rates seen in registrars (39\%) than in consultants $(25 \%),(P$ $=0.13)$ ) [75]. However, a study which compared sample adequacy between nurses (83.3\%) and doctors (80\%), $P>$ 0.05 , concluded that with the right training the ability to perform successful Pipelle is independent of professional category [77].

While few complications have been associated with Pipelle [73]. [78], mainly discomfort and false negative results, a study showed that Pipelle makes the endometrium approximately $0.4 \mathrm{~mm}$ thinner and creates echogenic spots which can be misinterpreted as sonographic lesions if the ultrasound is not performed prior to ES [79] (Table 1).

\section{Discussion}

Our aim was to search and synthesise the whole range of literature on ES in AUB in low-risk patients to guide further research and develop new evidence-based care pathways in primary care. Overall, the evidence that we have identified supports the use of ES in the outpatient setting and is a valuable source for the development of new care pathways in primary care.

To the best of our knowledge, this study is the first systematic review to primarily focus on the role of ES in assessing and managing AUB in low-risk women in the outpatient setting $[25,78,80-82]$. The available evidence shows that when Pipelle is combined with clinical assessment and ultrasound findings, it becomes a valuable tool for investigating AUB in low-risk women. Pipelle seems to perform as well or better than any other ES device in terms of sampling adequacy and sensitivity, with 
comparable results to D\&C which for years was the standard technique for obtaining endometrial tissue in patients with AUB [78]. Furthermore, Pipelle seems to be cost-effective and better tolerated in terms of pain/discomfort [83]. However, its use has shown to be limited by cervical stenosis, pelvic organ prolapse and endometrial atrophy $[24,75]$. Since Pipelle causes changes in the endometrium, it should not be performed before USS [79], and if the ultrasound reports localised lesions, a hospital referral for a hysteroscopy-guided biopsy may prove more useful than performing a blind Pipelle [84] given its limited sensitivity for focal lesions $[47,70]$.

Despite our robust and thorough literature search, we have noted some limitations in the available evidence. We only identified one study which was conducted on a primary care population by general practitioners [39] and one study which looked exclusively at premenopausal patients [40] and therefore, our conclusions are mainly based on studies which were carried out in either outpatient specialised clinics or hospital departments on a mixed cohort of pre- and postmenopausal women. Many of the studies that we identified were of poor or moderate methodological quality with wide-ranging inclusion and exclusion criteria (see Additional file 2). This heterogeneity may partly be responsible for the significant variability seen in terms of the sensitivity and specificity of Pipelle for detecting endometrial hyperplasia/cancer.

A meta-analysis was beyond the scope of this paper but critical appraisal and analysis of pooled data from diagnostic studies is an important next step in establishing the utility of ES. Given the limited information about the true test performance of ES in the community, it is not possible for clinicians to quantify the risk of hyperplasia/cancer (or other pathology) based only on ES. This is especially pertinent when the sample result is normal but the patient is still symptomatic; clinicians should then continue to consider the possibility of false negative results e.g. undiagnosed cancer/hyperplasia in these patients.

\section{Conclusions}

The evidence we analysed suggests that performing ES in the outpatient setting may allow effective management of low-risk women with AUB in primary care without referral to a hospital. But the false negative rate, health economics and implications of such a change in practice are still unknown and more research is required.

\section{Additional files}

Additional file 1: Literature search strategy. Search strategies and key words employed in the literature review. (DOCX $11 \mathrm{~kb}$ ).

Additional file 2: Study quality assessment. Assessment of methodological quality of the studies included in the literature review. (DOCX $62 \mathrm{~kb}$ ).

\section{Abbreviations}

AUB: Abnormal uterine bleeding; D\&C: Dilation and curettage;

ES: Endometrial sampling; PMB: Postmenopausal bleeding; RCT: Randomised controlled trial; USS: Ultrasound scan

\section{Acknowledgements}

We would like to thank Dr. Mary Connor and Dr. Mauro A Rinaldi for their valuable comments.

\section{Availability of data and materials}

All data generated or analysed during this study are included in this published article and its supplementary information files.

\section{Authors' contributions}

JMD proposed the review and created the original search strategy. BD undertook the initial literature search and retrieved the manuscripts. BN revised the initial search, executed the final searches, compiled the results and undertook the quality assessments. BN and JMD wrote the manuscript, with contributions from BD, which all authors approved. All authors read and approved the final manuscript.

Ethics approval and consent to participate Not required.

Consent for publication

Not applicable.

\section{Competing interests}

The authors declare that they have no competing interests.

\section{Publisher's Note}

Springer Nature remains neutral with regard to jurisdictional claims in published maps and institutional affiliations.

\section{Author details}

${ }^{1}$ Clinical Research Fellow in Obstetrics \& Gynaecology; Academic Unit of Reproductive and Developmental Unit, University of Sheffield, Sheffield S10 2SF, UK. ${ }^{2}$ Academic Unit of Primary Medical Care, University of Sheffield, Sheffield S5 7AU, UK.

Received: 27 September 2017 Accepted: 10 July 2018

Published online: 30 July 2018

\section{References}

1. Munro M, Critchley H, Broder M, Fraser ID. ftFWGoM. FIGO classification system (FIGO-COEIN) for causes of abnormal uterine bleeding in nongravid women of reproductive age. Int J Gynaecol Obstet. 2011;113(1):3-13.

2. Fraser I, Langham SU-HK. Health-related quality of life and economic burden of abnormal uterine bleeding. Expert Rev Obstet Gynecol. 2009:4:179-89.

3. Shapley M, Jordan KCP. An epidemiological survey of symptoms of menstrual loss in the community. Br J Gen Pract. 2004;54:359-63.

4. Garside R, Stein K, Wyatt K, Round APA. The effectiveness and costeffectiveness of microwave and thermal balloon endometrial ablation for heavy menstrual bleeding: a systematic review and economic modelling. Health Technol Assess (Rockv). 2004;8ii:155.

5. McCormick A, Fleming D, Charlton J, Royal College of General Practitioners GB S. OoPCa. Morbidity statistics from general practice: fourth national study. 1995.

6. Royal college of Obstetrics and Gynaecology. Heavy menstrual bleeding audit second annual report. 2012

7. Kai J, Middleton L, Daniels J, Pattison H, Tryposkiadis K, Gupta J, et al. Usual medical treatments or levonorgestrel-IUS for women with heavy menstrual bleeding: long-term randomised pragmatic trial in primary care. Br J Gen Pract. 2016;66(653):e861-e70. Epub 2016 Oct 10.

8. Dickson J, Delaney B, Connor M. Primary care endometrial sampling for abnormal uterine bleeding: a pilot study. J Fam Plan Reprod Heal Care. 2017:43:247-8.

9. Lethaby A, Cooke IRM. Progesterone or progestogen-releasing intrauterine systems for heavy menstrual bleeding. Cochrane Database Syst Rev. 2005;19:CD002126. 
10. Weeks A, Duffy SWJ. A double-blind randomised trial of leuprorelin acetate prior to hysterectomy for dysfunctional uterine bleeding. Br J Obstet Gynaecol. 2000;107:323-8.

11. Maresh M, Metcalfe M, McPherson K, Overton C, Hall V, Hargreaves J, et al. The VALUE national hysterectomy study: description of the patients and their surgery. Br J Obstet Gynaecol. 2002;109:302-12.

12. Pitkin J. Dysfunctional uterine bleeding. BMJ. 2007;334(7603):1110-1.

13. Warner P, Critchey H, Lumsden M, Cambell-Brown M, Dougas AMG. Referral for menstrual problems: cross sectional survey of symptoms, reasons for referral and management. BMJ. 2001;323:24-8.

14. Farquhar CM, Kimble RMN. How do New Zealand Gynaecologists treat menorrhagia? Aust New Zeal J Obstet Gynaecol. 1996;36:444-7. https://doi. org/10.1111/j.1479-828X.1996.tb02190.X.

15. Lumsden M, Gebbie AHC. Managing unscheduled bleeding in non-pregnant premenopausal women. BMJ. 2013;346. https://doi.org/10.1136/bmj.f3251.

16. Bignardi T, Van den Bosch T, Condous G. Abnormal uterine and postmenopausal bleeding in the acute gynaecology unit. Best Pract Res Clin Obstet Gynaecol. 2009;595-607. https://doi.org/10.1016/j.bpobgyn.2009.05.001.

17. Cancer Research UK. Uterine Cancer incidence statistics: uterine cancer incidence trends over time 2014. http://www.cancerresearchuk.org/healthprofessional/cancer-statistics/statistics-by-cancer-type/uterine-cancer/ incidence\#ref-2. (accessed 19 Jan 2017).

18. Wise MR, Gill P, Lensen S, Thompson JMD, Farquhar CM. Body mass index trumps age in decision for endometrial biopsy: cohort study of symptomatic premenopausal women. Am J Obstet Gynecol. 2016;215:598. e1-8. https://doi.org/10.1016/j.ajog.2016.06.006.

19. Cooper N, Barton P, Breijer M, Caffrey O, Opmeer B, Timmermans A, Cooper NA, Barton PM, Breijer M, Caffrey O, Opmeer BC. et al. Cost-effectiveness of diagnostic strategies for the management of abnormal uterine bleeding (heavy menstrual bleeding and post-menopausal bleeding): a decision analysis 2014;18. doi:https://doi.org/10.3310/hta18240.

20. National Insititute for Health and Care Excellence. Suspected cancer: recognition and referra. 2015.

21. Julian S, Naftalin N, Clark M, Szczepura A, Rashid A, Baker R, et al. An integrated care pathway for menorrhagia across the primary-secondary interface: patients' experience, clinical outcomes, and service utilisation. BMJ Qual Saf. 2007;16(2):110-5.

22. Royal College of Obstetrics and Gynaecology. Standards for Gynaecology. 2008.

23. Rauf RSA, Sadia S, Waqar F, Zafar S, Sultana SWS. Outpatient endometrial biopsy with Pipelle vs diagnostic dilatation and curettage. J Ayub Med Coll Abbottabad. 2004;26:145-8.

24. Fakhar S, Saeed G, Khan AH, Alam AY. Validity of pipelle endometrial sampling in patients with abnormal uterine bleeding. Ann Saudi Med. 2008;28:188-91.

25. Cooper JM, Erickson ML. Endometrial sampling techniques in the diagnosis of abnormal uterine bleeding. Obstet Gynecol Clin North Am. 2000;27:23544. https://doi.org/10.1016/S0889-8545(00)80018-2.

26. Stovall TG, Ling FW, Morgan PL. A prospective, randomized comparison of the Pipelle endometrial sampling device with the Novak curette. Am J Obstet Gynecol. 1991;165:1287-90. https://doi.org/10.1016/0002-9378(91)90351-Q.

27. Koonings $\mathrm{P}, \mathrm{Moyer} \mathrm{DL}$, Grimes DA. A randomised clinical trial comparing Pipelle and tis-u-trap for endometrial biopsy. Obstet Gynaecol. 1990;75:293.

28. Kaunitz AM, Masciello A, Ostrowski M, Rovira EZ. Comparison of endometrial biopsy with the endometrial Pipelle and Vabra aspirator. J Reprod Med. 1988;33:427-31.

29. Moberger B, Nilsson S, Palmstierna S, Redvall L, Sternby N. Amulticenter study comparing two endometrial sampling devices - Medscand Endorette TM and Pipelle De cornier R. Acta Obstet Gynecol Scand. 1998;77:764-9. https://doi.org/10.1034/j.1600-0412.1998.770712.x.

30. Williams ARW, Brechin S, Porter AJL, Warner P, Critchley HOD. Factors affecting adequacy of Pipelle and Tao brush endometrial sampling. BJOG An Int J Obstet Gynaecol. 2008;115:1028-36. https://doi.org/10. 1111/j.1471-0528.2008.01773.x.

31. Antoni J, Folch E, Costa J, Foradada CM, Cayuela E, Combalia N, et al. Comparison of cytospat and pipelle endometrial biopsy instruments. Eur J Obstet Gynecol Reprod Biol. 1997;72:57-61. https://doi.org/10. 1016/S0301-2115(96)02658-9.

32. Lipscomb GH, Lopatine SM, Stovall TG, Ling FW. A randomized comparison of the Pipelle, Accurette, and Explora endometrial sampling devices. Am J Obstet Gynecol. 1994;170:591-4. https://doi.org/10.1016/ S0002-9378(94)70234-9.
33. Glasziou P, Irwig L, Bain CCG. The question. In: Systematic Reviews in Health Care: a Practical Guide. New York: Cambridge University Press; 2001. p. 9.

34. Cornier E. The Pipelle: a disposable device for endometrial biopsy. Am J Obs Gynecol. 1984;148:109-10.

35. Cochrane Handbook for Systematic Reviews of Interventions | Cochrane Training n.d. http://training.cochrane.org/handbook (accessed 12 July 2017).

36. West S, King V, Carey TS, et al. Systems to Rate the Strength of Scientific Evidence: Summary. In: AHRQ Evidence Report Summaries. Rockville (MD): Agency for Healthcare Research and Quality (US); 1998-2005. 2002;47. Available from: https://www.ncbi.n/m.nih.gov/books/NBK11930/.

37. Macones G. How accurate if Pipelle sampling: a study by Huang et al. Am J Obstet Gynecol 2007;196(3):280-1.

38. McCluggage WG. My approach to the interpretation of endometrial biopsies and curettings. J Clin Pathol. 2006;59:801-12. https://doi.org/10. 1136/jcp.2005.029702.

39. Seamark CJ. Endometrial sampling in general practice. Br J Gen Pract [Internet]. 1998;48:434. Available from: http://bjgp.org/content/48/434/1597/ tab-pdf

40. Henig I, Tredway DR, Maw GM, Gullett AJ, Cheatwood M, C P. Evaluation of the Pipelle curette for endometrial biopsy. J Reprod Med. 1989;34:786-9.

41. Zorlu CG, Cobanoglu O, Işik AZ, Kutluay L, Kuşçu E. Accuracy of Pipelle endometrial sampling in endometrial carcinoma. Gynecol Obstet Investig. 1994:38:272-5.

42. Ferry J, Farnsworth A, Webster M, Wren B. The efficacy of the Pipelle endometrial biopsy in detecting endometrial carcinoma. Aust New Zeal J Obstet Gynaecol. 1993;33:76-8. https://doi.org/10.1111/j.1479-828X.1993.tb02060.X.

43. Sany $\mathrm{O}$, Singh $\mathrm{K}$, Jha S. Correlation between preoperative endometrial sampling and final endometrial cancer histology. Eur J Gynaecol Oncol. 2012;33:142-4.

44. Huang GS, Gebb JS, Einstein MH, Shahabi S, Novetsky AP, Goldberg GL. Accuracy of preoperative endometrial sampling for the detection of highgrade endometrial tumors. Am J Obstet Gynecol. 2007;196:243.e1-5. https:// doi.org/10.1016/j.ajog.2006.09.035.

45. Daud S, Jalil SSA, Griffin M, Ewies AAA. Endometrial hyperplasia - the dilemma of management remains: a retrospective observational study of 280 women. Eur J Obstet Gynecol Reprod Biol. 2011;159:172-5. https://doi. org/10.1016/j.ejogrb.2011.06.023.

46. Liu H, Wang FL, Zhao YM, Yao YQ, Li YL. Comparison of Pipelle sampler with conventional dilatation and curettage (D\&C) for Chinese endometrial biopsy. J Obstet Gynaecol. 2015;35:508-11.

47. Gungorduk K, Asicioglu O, Ertas IE, Ozdemir LA, Ulker MM, Yildirim G, et al. Comparison of the histopathological diagnoses of preoperative dilatation and curettage and pipelle biopsy. Eur J Gynaecol Oncol. 2014;35:539-43. https://doi.org/10.12892/ejgot24972014.

48. Kazandi M, Okmen F, Ergenoglu AM, Yeniel AO, Zeybek B, Zekioglu O, et al. Comparison of the success of histopathological diagnosis with dilatationcurettage and Pipelle endometrial sampling. J Obstet Gynaecol. 2012;32: 790-4. https://doi.org/10.3109/01443615.2012.719944.

49. Demirkiran F, Yavuz E, Erenel H, Bese T, Arvas M, Sanioglu C. Which is the best technique for endometrial sampling? Aspiration (pipelle) versus dilatation and curettage (D\&C). Arch Gynecol Obstet. 2012;286:1277-82. https://doi.org/10.1007/s00404-012-2438-8.

50. Ben-Baruch G, Seidman DS, Schiff E, Moran O, Menczer J. Outpatient endometrial sampling with the Pipelle curette. Gynecol Obstet Investig. 1994;37:260-2.

51. Kavak Z, Ceyhan N, Pekin S. Combination of vaginal ultrasonography and pipelle sampling in the diagnosis of endometrial disease. Aust NZ Obs Gynaecol. 1996;36:63.

52. Abdelazim IA, Elezz AA, Abdelkarim AF. Pipelle endometrial sampling versus conventional dilatation \&amp; curettage in patients with abnormal uterine bleeding. Asian Pacific J Reprod. 2013;2:45-8. https:// doi.org/10.1016/S2305-0500(13)60115-3.

53. Rezk M, Sayyed T, Dawood R. The effectiveness and acceptability of Pipelle endometrial sampling versus classical dilatation and curettage: a three-year observational study. Gynecol Obstet Investig. 2016;81:537-42. https://doi.org/10.1159/000444711

54. Eddowes HA, Read MD, Codling BW. Pipelle: a more acceptable technique for outpatient endometrial biopsy. BJOG An Int J Obstet Gynaecol. 1990;97: 961-2. https://doi.org/10.1111/j.1471-0528.1990.tb02458.x.

55. Naim NM, Ahmad S, Razi ZR, M ZA. The Vabra aspirator versus the Pipelle device for outpatient endometrial sampling. Aust N Z J Obs Gynaecol. 2007;47:132-6. 
56. Rodriguez GC, Yaqub N, King ME. A comparison of the Pipelle device and the Vabra aspirator as measured by endometrial denudation in hysterectomy specimens: The Pipelle device samples significantly less of the endometrial surface than the Vabra aspirator. Am J Obstet Gynecol. 1993; 168:55-9. https://doi.org/10.1016/S0002-9378(12)90884-4.

57. Critchley HOD, Warner P, Lee AJ, Brechin S, Guise J, Graham B. Evaluation of abnormal uterine bleeding: comparison of three outpatient procedures within cohorts defined by age and menopausal status. Health Technol Assess. 2004;8(34):8.

58. Yang GC, Del Priore G, W LS. Factors influencing the detection of uterine cancer by suction curettage and endometrial brushing. J Reprod Med. 2003;47:1005-10.

59. Del Priore G, Williams R, Harbatkin CB, Wan LS, Mittal K, Yang GCH. Endometrial brush biopsy for the diagnosis of endometrial Cancer. Obstet Gynecol Surv. 2001;56:548-9.

60. Yang GC, Wan LS. Endometrial biopsy using the Tao brush method. A study of 50 women in a general gynecologic practice. J Reprod Med. 2000;45:109-14.

61. Silver MM, Miles P, Rosa C. Comparison of Novak and Pipelle endometrial biopsy instruments. Obstet Gynecol. 1991;78:828-30.

62. Leclair CM, Zia JK, Doom CM, Morgan TK, Edelman AB. Pain experienced using two different methods of endometrial biopsy: a randomized controlled trial. Obstet Gynecol. 2011;117:636-41. https://doi.org/10.1097/ AOG.0b013e31820ad45b.

63. Bhide A, Gangji A, Anyanwu L. Endometrial biopsy: A pilot study of instrument used; Pipelle vs infant feeding tube. J Obstet Gynaecol. 2007;27: 838-9. https://doi.org/10.1080/01443610701718941.

64. Masood H, Ashraf S, Masood MS. Frequency of positive endometrial pipelle biopsies in patients with abnormal uterine bleeding for detection of endometrial carcinoma Pak J Med Health Sci. 2015;9:256-8. https://pdfs. semanticscholar.org/c724/5b497b4f5771a8e4356db03be05fc8a0f760.pdf. Accessed Apr 2017.

65. Piatek S, Panek G, Wielgoś M. Assessment of the usefulness of pipelle biopsy in gynecological diagnostics. Ginekol Pol. 2016;87:559-64. https://doi. org/10.5603/GP.2016.0044.

66. Machado F, Moreno J, Carazo M, León J, Fiol G, Serna R. Accuracy of endometrial biopsy with the cornier pipelle for diagnosis of endometrial cancer and atypical hyperplasia. Eur J Gynaecol Oncol. 2003;24:279-81.

67. Guido RS, Kanbour-Shakir A, Rulin MC, Christopherson WA. Pipelle endometrial sampling. Sensitivity in the detection of endometrial cancer. J Reprod Med. 1995;40:553-5.

68. Sanam M, Majid M. Comparison the diagnostic value of dilatation and curettage versus endometrial biopsy by Pipelle--a clinical trial. Asian Pac J Cancer Prev. 2015;16:4971-5. https://doi.org/10.7314/APJCP.2015.16.12.4971.

69. Goldchmit R, Katz Z, Blickstein I, Caspi B, Dgani R. The accuracy of endometrial Pipelle sampling with and without sonographic measurement of endometrial thickness. Obstet Gynecol. 1993;82:727-30.

70. Seto MTY, Ip PPC, Ngu S-F, Cheung ANY, Pun T-C. Positive predictive value of endometrial polyps in Pipelle aspiration sampling: a histopathological study of 195 cases. Eur J Obstet Gynecol Reprod Biol. 2016;203:12-5. https:// doi.org/10.1016/j.ejogrb.2016.04.027.

71. Polena V, Mergui J-L, Zerat L, Sananes S. The role of Pipelle ${ }^{\circledR}$ Mark II sampling in endometrial disease diagnosis. Eur J Obstet Gynecol Reprod Biol. 2007;134:233-7. https://doi.org/10.1016/j.ejogrb.2006.07.026.

72. Shams, G. Comparison of Pipelle de Cornier with conventional dilatation and curettage in terms of patients' acceptability. Journal of Postgraduate Medical Institute ;Peshawar. 2012: 26 (4) [cited 2017 Jun 22]. Available from: http://www.jpmi.org.pk/index.php/jpmi/article/view/1377

73. Trolice MP, Fishburne CJR, McGrady S. Anesthetic efficacy of intrauterine lidocaine for endometrial biopsy: a randomized double-masked trial. Obstet Gynecol. 2000;95:345-7.

74. Frishman G, Jacobs S. A randomized clinical trial comparing Pipelle and tisU-trap for endometrial biopsy. Obstet Gynecol. 1990;76:315-6.

75. Gordon SJ, Westgate J. The incidence and Management of Failed Pipelle Sampling in a general outpatient clinic. Aust New Zeal J Obstet Gynaecol. 1999;39:115-8. https://doi.org/10.1111/j.1479-828X.1999.tb03460.X.

76. Brandner P, Neis KJ. Diagnosis of endometrial Cancer and its precursors. Contrib Gynecol Obstet. 2000;20:27-40.

77. Dantas MC, Hidalgo MM, Bahamondes L, Marchi NM. A comparison of the performance of endometrial biopsy with the Pipelle ${ }^{\odot}$ by nurses and physicians. Int J Gynecol Obstet. 1994;45:164-5. https://doi.org/10.1016/ 0020-7292(94)90128-7.
78. Youssif SN, McMillan DL. Outpatient endometrial biopsy: the pipelle. Br J Hosp Med. 1995;54(5):198-201.

79. Van den Bosch T, Van Schoubroeck D, Ameye L, Van Huffel S, Timmerman D. Ultrasound examination of the endometrium before and after Pipelle endometrial sampling. Ultrasound Obstet Gynecol. 2005;26: 283-6. https://doi.org/10.1002/uog.1967.

80. Dijkhuizen FPHLJ, Mol BWJ, Brölmann HAM, Heintz APM. The accuracy of endometrial sampling in the diagnosis of patients with endometrial carcinoma and hyperplasia. Cancer. 2000;89:1765-72. https://doi.org/10. 1002/1097-0142(20001015)89:8<1765::AID-CNCR17>3.0.CO;2-F.

81. Du J, Li Y, Lv S, Wang Q, Sun C, Dong $X$, et al. Endometrial sampling devices for early diagnosis of endometrial lesions. J Cancer Res Clin Oncol. 2016;142: 2515-22. https://doi.org/10.1007/s00432-016-2215-3.

82. Clark TJ, Mann CH, Shah N, Khan KS, Song F, Gupta JK. Accuracy of outpatient endometrial biopsy in the diagnosis of endometrial hyperplasia. Acta Obstet Gynecol Scand. 2001;80:784-93.

83. Chambers JT, Chambers SK. Endometrial sampling: when? Where? Why? With what? Clin Obstet Gynecol. 1992;35:28-39.

84. Kotdawala P, Kotdawala S, Nagar N. Evaluation of endometrium in perimenopausal abnormal uterine bleeding. J Midlife Health. 2013;4:16-21. https://doi.org/10.4103/0976-7800.109628.

85. Tahir MM, Bigrigg MA, Browning JJ, Brookes T, Smith PA. A randomised controlled trial comparing transvaginal ultrasound, outpatient hysteroscopy and endometrial biopsy with inpatient hysteroscopy and curettage. BJOG An Int J Obstet Gynaecol. 1999;106:1259-64. https://doi. org/10.1111/j.1471-0528.1999.tb08179.x.

86. Ahonkallio SJ, Liakka AK, Martikainen HK, Santala MJ. Feasibility of endometrial assessment after thermal ablation. Eur J Obstet Gynecol Reprod Biol. 2009;147:69-71. https://doi.org/10.1016/j.ejogrb.2009.06.014.

\section{Ready to submit your research? Choose BMC and benefit from:}

- fast, convenient online submission

- thorough peer review by experienced researchers in your field

- rapid publication on acceptance

- support for research data, including large and complex data types

- gold Open Access which fosters wider collaboration and increased citations

- maximum visibility for your research: over $100 \mathrm{M}$ website views per year

At BMC, research is always in progress.

Learn more biomedcentral.com/submissions 